\title{
Millimole Per Gram of Creatinine
}

National Cancer Institute

\section{Source}

National Cancer Institute. Millimole Per Gram of Creatinine. NCI Thesaurus. Code

C105510.

A unit of substance concentration defined as the concentration of millimoles of solute per gram of creatinine. 